\title{
Dysmorphic syndromes with demonstrable biochemical abnormalities
}

\author{
P T CLAYTON AND ELIZABETH THOMPSON \\ From the Departments of Child Health and Clinical Genetics, Institute of Child Health, London WC1N 1 EH.
}

SUMMARY Many inborn errors of metabolism are associated with dysmorphic manifestations. In this review, we have attempted to correlate the dysmorphic features with the underlying metabolic defect or its consequences. Most of the defects which we have discussed affect the synthesis or degradation of macromolecules (for example, collagen, elastin, bone mineral, proteoglycans, glycoproteins, and triglycerides). Such defects may affect either a single enzyme or multiple enzymes in specific organelles, such as lysosomes or peroxisomes, or they may affect hormonal control of synthesis and degradation. Examples are also included of defects affecting the catabolism of simple molecules when accumulating metabolites have a secondary effect on macromolecules, as in homocystinuria. In a number of instances, however, the correlation between the biochemical abnormality and the dysmorphic features are not understood. Ultimately, all dysmorphic syndromes will be attributable to a biochemical defect or its effects. The aim of this overview is to provide an insight into the relationship between the two at the present time.

The study of inborn errors of metabolism has made substantial contributions to our understanding of biochemistry. It is worthwhile, therefore, to consider whether we can learn anything about the biochemistry of morphogenesis and dysmorphogenesis from studies of those inborn errors which produce dysmorphic features. The first conclusion to be drawn from a survey of inborn errors ${ }^{12}$ is that, as a general rule, dysmorphic manifestations can be expected as a result of disorders which affect the synthesis or early steps in the degradation of the large molecules which form the structural framework of cells and extracellular matrix. Many of these macromolecular reactions are under hormonal control and therefore disorders affecting the synthesis and biological action of hormones may also lead to dysmorphic features. Inborn errors affecting the metabolism of simple molecules (for example, organic acidaemias) do not usually lead to dysmorphic features unless there are secondary effects on macromolecules.

Major components of the extracellular matrix include collagen, proteoglycans $(95 \%$ polysaccharide, $5 \%$ protein), glycoproteins, elastin, and bone mineral. The architecture of cells is determined by cell membranes and by intracellular structural pro-

Received for publication 5 December 1987 Accepted for publication 10 December 1987. teins and also by their content of stored macromolecules (for example, triglyceride in normal adipocytes or intralysosomal material in lysosomal storage disorders). Cell membranes are composed largely of phospholipids (phosphoglycerides, sphingomyelin, and plasmalogens), glycolipids, and cholesterol. Disorders affecting the metabolism of some of the macromolecules listed above are well known; others have only recently been recognised.

\section{Disorders of collagen and elastin metabolism}

Collagen is the major fibrous element of skin, bone, tendon, and cartilage and is responsible for their tensile strength. Bone contains mainly type I collagen whereas dermal collagen is approximately

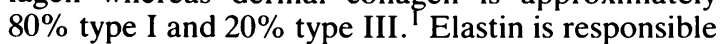
for the elastic properties of the skin and of the walls of blood vessels.

It is now generally accepted that defective synthesis of type I collagen can result in weakness of the bones with consequent fractures and deformity: osteogenesis imperfecta (OI). A variety of mutaions in the chains of type I collagen have been described in autosomal dominant and recessive forms of the disease. ${ }^{3}$ Type I collagen is a polymer, assembled from $\alpha 1(\mathrm{I})$ and $\alpha 2(\mathrm{I})$ chains in the ratio of $2: 1$. Sykes $^{4}$ has suggested that mutant $\alpha$ chains which become included in the collagen molecule 
may produce more severe phenotypic effects than those in which the mutation prevents the $\alpha$ chain from being incorporated into the collagen molecule. $\mathrm{OI}$ is not always caused by a mutation in one of the structural genes for type I collagen (COLIA1 and COLIA2); another postulated cause is a recessively inherited defect in one of the enzymes required for collagen production. ${ }^{5}$ Equally, defective synthesis of type I collagen does not always produce osteogenesis imperfecta; it may produce severe joint laxity with multiple dislocations and subluxations: EhlersDanlos syndrome (EDS) type VII. The common biochemical feature in patients with EDS type VII appears to be impaired removal of the amino terminal $\mathrm{N}$-propeptides from procollagen. The documented defects include heterozygous structural alterations of the proa1(I) or proa1(II) chains which prevent cleavage of the abnormal chain by procollagen $\mathrm{N}$-proteinase. ${ }^{6-10}$ It is possible that a similar clinical picture may be caused by deficiency of the proteinase itself, inherited in an autosomal recessive manner (McKusick 22541 11 ).

Defective synthesis of type III collagen can reduce the tensile strength of the skin and joints and so this can also cause Ehlers-Danlos syndrome, in this case the severe ecchymotic form (EDS type IV). ${ }^{12} 13$

The synthesis of both type I and type III collagen requires post-translational modification of the procollagen by conversion of lysine residues to hydroxylysine. Lysyl hydroxylase deficiency causes the autosomal recessive ocular-scoliotic form of EDS (type VI). ${ }^{13} 14$

Impaired synthesis or increased catabolism of elastin reduces the elasticity of the skin and can therefore produce cutis laxa. Anderson et al $^{15}$ have described an autosomal recessive form of cutis laxa with pulmonary emphysema associated with high serum activity of an elastase. ${ }^{15}$

Cross links between individual collagen molecules and between individual elastin molecules are forged by lysyl oxidase, a copper dependent enzyme. In X linked cutis laxa (previously called EDS type IX), Byers et $a l^{16}$ reported low levels of lysyl oxidase activity and low serum copper and caeruloplasmin levels. Clinical features included moderate skin laxity, hyperextensible skin, mild joint laxity, dilatations of the urinary tract, and inguinal herniae. In Menkes syndrome, ${ }^{1}$ the disordered copper metabolism produces severe disruption of the elastic tissue of blood vessels and emphysema and bladder diverticula have also been described. Abnormalities of skin elastin are detectable on electron microscopy: these ultimately lead to cutis laxa and may perhaps contribute to the pudgy cheeks. In cartilage, irregularity of collagen fibrils has been described and the bones are osteoporotic. The most striking dysmorphic feature of Menkes syndrome, the steely hair, is not attributable to lysyl oxidase deficiency. Danks has suggested that it may be the result of deficiency of another copper dependent enzyme that cross links keratin by forming disulphide bonds. ${ }^{1}$

In cystathionine synthetase deficiency, ${ }^{\prime}$ accumulation of homocysteine impairs the formation of the cross links between lysyl and hydroxylysyl residues of collagen and this is thought to be responsible for some of the dysmorphic features, for example, osteoporosis and joint laxity. However, the highly characteristic lens dislocation is more likely to result from disruption of the disulphide bonds of another fibrous protein as a result of homocysteine accumulation. Lens dislocation also occurs in sulphite oxidase deficiency ${ }^{2}$ and molybdenum cofactor deficiency ${ }^{17}$; in these conditions it is thought to be the sulphite accumulation which disrupts the disulphide bonds.

\section{Disorders affecting the bone mineral}

Failure to deposit bone mineral in the matrix of osteoid and epiphyseal cartilage leads to rickets, with beading of the costrochondral junctions, bowing of the legs, and widening of the metaphyses of the long bones. Such changes can be the result of inborn errors affecting renal tubular reabsorption of phosphate, conversion of vitamin $D$ to active metabolites, or end organ sensitivity to these metabolites. ${ }^{1}$ Failure of mineralisation also occurs in hypophosphatasia, ${ }^{1}$ which is characterised by low activities of alkaline phosphatase in bone and serum and by increased urinary excretion of phosphoethanolamine and inorganic pyrophosphate. The failure of mineralisation is probably caused by the reduced activity of alkaline phosphatase in the bone. This prevents hydrolysis of pyrophosphate and other phosphate esters. Hence, the accumulation of inorganic phosphate in matrix vesicles, which would normally lead to the deposition of bone mineral, is impaired. The dysmorphic features of hypophosphatasia include not only the signs of rickets but also skin dimples and, in some cases, gross craniofacial dysmorphism as a result of premature synostosis of skull bones. Synostosis occurs because, in the uncalcified osteoid, the fibrous septa that normally separate the individual bones fail to develop. The same phenomenon occurs in acrocephalosyndactyly, but the cause of delayed calcification of osteoid in Apert and related syndromes is not known.

Normal bone growth involves remodelling and therefore catabolism of bone mineral. This process, as well as mobilisation of calcium to restore the plasma calcium concentration, is controlled by parathormone. Pseudohypoparathyroidism ${ }^{1}$ is caused 
by target organ resistance to parathormone. Hypocalcaemia is usual and there is a reduced urinary excretion of cyclic AMP in response to parathormone. Most patients are of short stature and have a round face with a depressed nasal bridge and multiple discrete abnormalities in the bones, including short metacarpals and metatarsals. The latter defects arise as a result of premature closure of the epiphyses preceded by a decrease in longitudinal growth. Reduced bone growth is a predictable consequence of impaired remodelling, but why it should affect the fourth and fifth digits and spare the others is difficult to explain.

\section{Disorders affecting subcutaneous fat}

Insulin stimulates synthesis of triglyceride and inhibits lipolysis in adipocytes. A reduction in the amount of fat in the subcutaneous tissues is responsible for some of the dysmorphic features in leprechaunism, generalised lipodystrophy, and partial lipodystrophy. Elsas et $a l^{18}$ have shown that leprechaunism can occur as the result of a deficiency of high affinity insulin receptors. In generalised lipodystrophy, hyperinsulinism and insulin resistance may also be found but the cause of the syndrome is not fully understood. Partial lipodystrophy has been recorded in association with familial deficiency of the $\mathrm{C} 3$ component of complement and loss of adipose tissue may have an immunological basis in this instance. ${ }^{19}$

\section{Lysosomal storage disorders ${ }^{12} 20$}

The lysosomes are the site of catabolism of many structural macromolecules, including glycosaminoglycans (mucopolysaccharides) derived from proteoglycans, glycoproteins, sphingolipids (sphingomyelin and ceramide), and glyco(sphingo)lipids (for example, gangliosides). In some lysosomal storage disorders, catabolism of only one type of compound is impaired, for example, the glycosaminoglycan dermatan sulphate in Maroteaux-Lamy disease (aryl sulphatase B deficiency). However, some lysosomal enzymes catalyse the hydrolysis of more than one type of macromolecule and so, for example, in infantile GM1 gangliosidosis, deficiency of $\beta$ galactosidase $A$ leads to reduced catabolism of gangliosides, glycosaminoglycans, and glycoproteins. In other lysosomal storage disorders the primary defect leads to reduced activity of more than one enzyme (for example, multiple sulphatase deficiency, I cell disease). Finally, the distension of lysosomes with undigested macromolecules may lead to secondary effects on the catabolism of other macromolecules. Nevertheless, it is possible to dissect out the major effects of the primary defects.
The effect of a block of sphingolipid or glycosphingolipid catabolism depends upon the localisation and function of the lipid. Galactocerebrosides and their sulphates are lipids of the central nervous system and so impaired degradation leads to neurological disease rather than dysmorphic features (for example, Krabbé disease, metachromatic leucodystrophy). Gangliosides are also found mainly in the CNS and in GM2 gangliosidoses (Tay Sachs disease and Sandhoff disease) CNS symptoms predominate. However, accumulation of gangliosides and gliosis can lead to macrocephaly and, in addition, many patients have doll-like facies with pale translucent skin, long eyelashes, fine hair, and delicate pink colouring. Whether these dysmorphic features are caused by ganglioside accumulation is uncertain; histochemical techniques reveal only limited lipid storage outside the CNS. GM1 gangliosidosis can occur as a neurological disease without obvious dysmorphic features but this is not the case for the infantile form (see below). Glucocerebrosides, neutral glycosphingolipids, sphingomyelin, and ceramide are widely distributed outside the CNS as constituents of cell membranes. Accumulation of glucocerebrosides can produce bony deformity (Gaucher disease), accumulation of neutral glycosphingolipids (in particular trihexosylceramide) produces corneal dystrophy, cataracts, and angiokeratoma corporis diffusum (Fabry disease), and accumulation of ceramide causes a tissue reaction which leads to swollen joints and periarticular nodules (Farber disease).

The effect of a block in the pathway for degradation of one of the glycosaminoglycans depends upon the function of the parent proteoglycan. Proteoglycans containing keratan sulphate are important constituents of the extracellular matrix of cartilage and the cornea. Remodelling of bones and joints during growth is dependent upon turnover of the cartilage matrix. Impaired degradation of keratan sulphate in Morquio disease (types $\mathbf{A}$ and $\mathbf{B}$ ) is associated with severe dysostosis multiplex, growth retardation, joint laxity, and corneal clouding.

Proteoglycans containing dermatan sulphate are important constituents of the extracellular matrix of interstitial connective tissue. Impaired degradation of dermatan sulphate in Maroteaux-Lamy disease, Hurler disease, and Hunter disease is associated with thickening of subcutaneous tissues producing a coarse facies, thick lips and gum ridges, and herniae. Increased stiffness of ligaments and other connective tissues leads to deformities of joints such as claw hand. Dermatan sulphate is not a major component of cartilage and yet chondrocytes do become vacuolated and irregularly arranged in the growing areas of bones and this is probably the basis of the 
dysostosis multiplex (which is milder than that of Morquio disease). In the cornea, dermatan sulphate occurs as a mixed chondroitin-dermatan sulphate proteoglycan. Degradation of this proteoglycan requires $\alpha$-L-iduronidase and arylsulphatase $B$ but is much less dependent upon iduronate sulphatase, either because of undersulphation or because of an easier bypass of the block by hyaluronidase. Hence, corneal clouding occurs in Hurler disease (iduronidase deficiency) and Maroteaux-Lamy disease (arylsulphatase B deficiency), but not in Hunter disease (iduronate sulphatase deficiency).

Proteoglycans containing heparan sulphate are not components of the extracellular matrix; they are components of cell membranes including the limiting membranes of synaptosomes in the CNS. Impaired degradation of heparan sulphate is the common feature in patients with Sanfilippo disease types $\mathrm{A}$ to $\mathrm{D}$. These disorders are characterised by mental retardation and regression but only mild dysmorphic features (coarse facies and dysostosis multiplex).

Glycoproteins occur widely within cells, on the cell surface, and extracellularly both within and without the CNS. The effects of impaired glycoprotein catabolism can be identified in $\alpha$ mannosidosis and aspartylglycosaminuria. (In other glycoproteinoses there is also impaired degradation of glycolipids and perhaps glycosaminoglycans.) In both $\alpha$ mannosidosis and aspartylglycosaminuria, facial coarsening, herniae, opacities of the lens or cornea or both, and a variable degree of dysostosis multiplex occur. Thus, the dysmorphic effects of impaired glycoprotein catabolism appear to be similar to those of impaired dermatan sulphate catabolism. In fucosidosis type 2 and sialidosis type 2 (juvenile type ${ }^{1}$ ), these dysmorphic features occur together with angiokeratomata, similar to those seen in Fabry disease. In fucosidosis this can be explained by the defect in glycolipid catabolism which leads to the accumulation of a pentaglycosylceramide which is closely related to the glycosylceramides that accumulate in Fabry disease. Early reports of human $\beta$ mannosidosis ${ }^{21}$ suggest that coarse features and skeletal abnormalities do not occur, but angiokeratomata can. This is very difficult to explain as the defect should affect glycoprotein degradation rather than glycolipid degradation! One patient who was deficient in both $\beta$ mannosidase and heparan- $\mathrm{N}$-sulphatase (the enzyme which is deficient in Sanfilippo disease type A) did have both coarse features and bony abnormalities. ${ }^{22}$

In infantile GM1 gangliosidosis, catabolism of gangliosides, keratan sulphate, and glycoproteins are all severely impaired as a result of a defect of $\beta$ galactosidase A. Hence, severe neurological disease coexists with major skeletal abnormalities and $\stackrel{\vec{S}}{\rightarrow}$ coarse features. In I cell disease and pseudo-Hurler $\bar{C}$ polydystrophy, there is a defect in the post-translational modification of a number of lysosomal acid $\frac{\bar{\omega}}{7}$ hydrolases. The mannose residues of these glyco- $\stackrel{\mathbb{D}}{\stackrel{D}{2}}$ protein enzymes are not phosphorylated normally, क which impairs translocation into the lysosomes and leads to further glycosylation of the enzymes. The ? phenotypic expression of these disorders appears to $\overrightarrow{\vec{\omega}}$ be affected by the fact that impaired lysosomal $\stackrel{\omega}{\omega}$ function, as indicated by their distension (presumably with undigested macromolecules), occurs largely in connective tissue cells. The dysmorphic features of resemble those seen in disorders of dermatan sulphate or glycoprotein degradation, but there is no के corneal clouding. In multiple sulphatase deficiency $\circ$ there is impaired catabolism of keratan sulphate and dermatan sulphate and consequent dysostosis multi- $\vec{c}$ plex and coarse features. In addition, however, there is ichthyosis as a result of steroid sulphatase deficiency.

The above survey indicates that in the lysosomal storage disorders, dysmorphic features occur largely as a result of impaired turnover of structural macromolecules within cells and in the extracellular matrix. This results in progressive distortion of the tissue and may provoke a tissue reaction such as $\frac{\mathbb{D}}{\varnothing}$ granuloma formation or gliosis. In many cases the $\overrightarrow{\vec{P}}$ dysmorphic features are not present at birth but become evident during childhood.

The investigations which may be helpful in a patient who has or is developing coarse facial features are shown in table 1 . There are a number of inborn errors of metabolism affecting either the synthesis of thyroid hormones or their effect on 3 . target organs ${ }^{1}$ and the hypothyroidism which results $\dot{\delta}$ may produce coarsening of facial features, beaking $₹$ of lumbar vertebrae, and epiphyseal dysgenesis; thyroid hormones are essential for the normal turnover of the extracellular matrix.

\section{Peroxisomal disorders}

Peroxisomes are round or ovoid subcellular N organelles slightly smaller than mitochondria and with a single limiting membrane and no cristae. They can be shown histochemically by making use of their peroxidase activity. Over the last few years the number of genetic disorders attributable to malfunc- 0 tioning of peroxisomal enzyme systems has been ${ }_{0}^{\circ}$

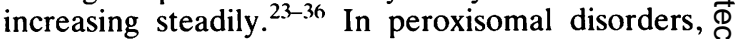
the dysmorphic features are well established at birth $\vec{\nabla}$ and thus the biochemical abnormalities must be $\frac{\varrho}{\sigma}$ exerting their influence in utero. Peroxisomal defects vary from those in which a single peroxisomal 
enzyme is deficient to those in which the whole organelle is absent. Thus, it is possible to build up a picture of the consequences of a single metabolic block and of combinations of interrupted pathways. The investigations which can be used to define the nature of a peroxisomal disorder are summarised in table 2 . Some of the reactions occurring within the peroxisome are concerned with the catabolism of small molecules, for example, hydrogen peroxide ${ }^{23}$ and glyoxylate, ${ }^{24}$ and inborn errors of metabolism

TABLE 1 Investigations which may aid in the diagnosis of a patient who has or is developing coarse facial features.

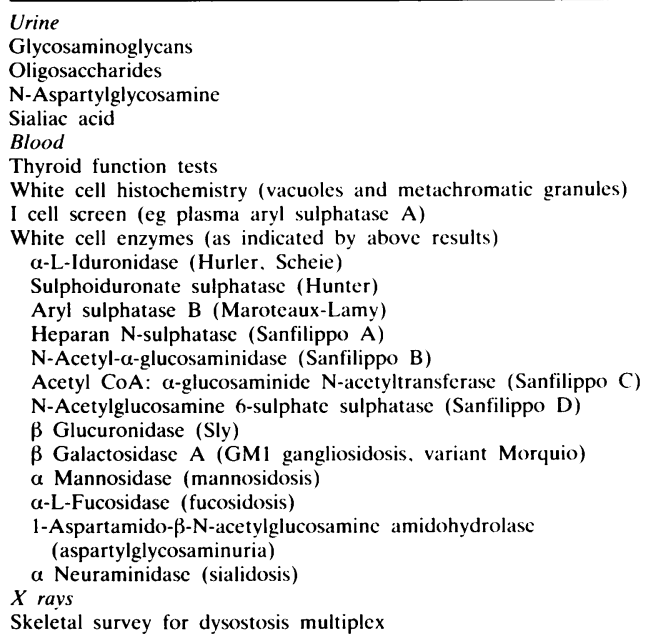

TABLE 2 Investigations used to delineate the nature of a peroxisomopathy.

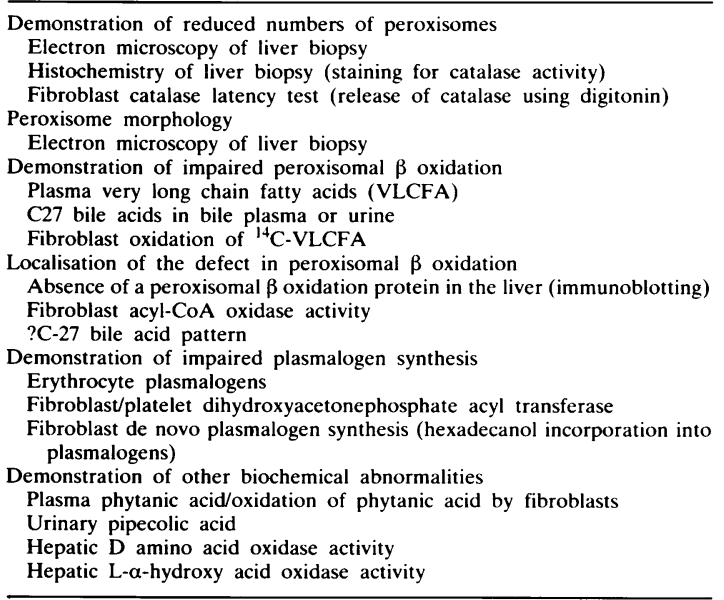

affecting these reactions do not produce dysmorphic features. However, peroxisomes are also responsible for synthesis of certain phospholipids (plasmalogens) and for the $\beta$ oxidation of fatty acids. Plasmalogens are important constituents of cell membranes and the acetyl-CoA generated by peroxisomal $\beta$ oxidation is used in the synthesis of cholesterol and for other syntheses involving acetylation reactions. ${ }^{25}$

The peroxisomal pathway for $\beta$ oxidation of coenzyme A esters of fatty acids involves, sequentially, acyl-CoA oxidase, a bifunctional protein with enoyl-CoA hydratase and 3-hydroxyacyl-CoA oxidase activities, and 3-oxoacyl-CoA thiolase. In addition, oxidation of very long chain fatty acids (VLCFA) requires a VLCFA-CoA synthetase. Deficiency of the peroxisomal VLCFA-CoA synthetase produces $\mathrm{X}$ linked adrenoleucodystrophy and its variants. $^{26} 27$ These patients have no dysmorphic features despite the fact that accumulation of VLCFA can be shown in the fetus; adrenal atrophy and demyelination first produce symptoms at seven to eight years. Patients with a deficiency of one of the $\beta$ oxidation enzymes (acyl-CoA oxidase ${ }^{28}$ or 3-oxoacyl-CoA thiolase ${ }^{29-31}$ ) do not have striking dysmorphic features either; the mild dysmorphic features can probably be attributed to the severe hypotonia of prenatal onset (expressionless face, tented mouth, high arched palate, bell shaped chest, single transverse palmar creases) and delayed osseous maturation (large fontanelle). However, there are developmental anomalies in these patients. The hypotonia is in part the result of abnormalities of the cerebellum which shows demyelination and gliosis but also neuronal heterotopias, indicating defective neuronal migration. Renal cortical cysts may also be present. Thus, deficiency of a single peroxisomal $\beta$ oxidation enzyme can cause abnormalities of organogenesis.

Defects in the peroxisomal pathway for the synthesis of either phospholipids (plasmalogens) have been described in both rhizomelic chondrodysplasia punctata ${ }^{32}$ and Conradi-Hünermann syndrome. ${ }^{33}$ The enzyme catalysing the first step, dihydroxyacetone phosphate acyl transferase (DHAPAT), is deficient in skin fibroblasts in both conditions. Thus, it is tempting to postulate that deficient plasmalogen synthesis can give rise to punctate epiphyseal mineralisation with associated limb shortening and joint contractures, a prominent forehead, a low, flat nasal bridge, a short nose with anteverted nostrils, malar hypoplasia, cataracts, a short neck, and ichthyosis (fig 1). In rhizomelic chondrodysplasia punctata, which is inherited as an autosomal recessive trait, all cells express the defect so that the abnormalities are severe and symmetrical. In the $\mathrm{X}$ linked dominant form of Conradi- 


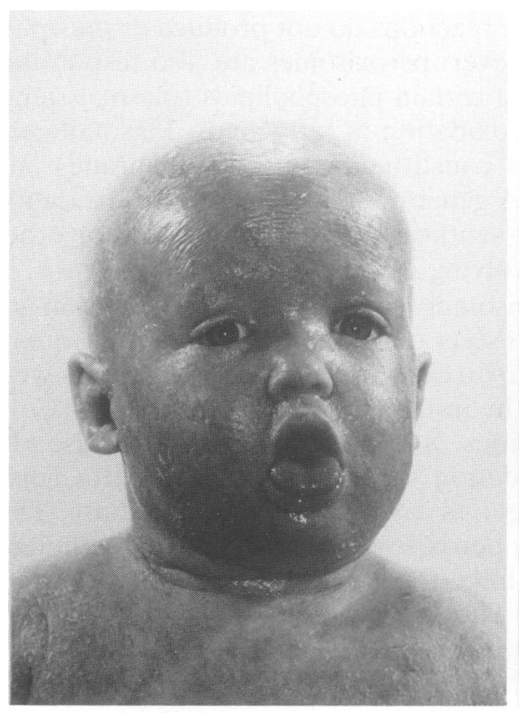

(a)

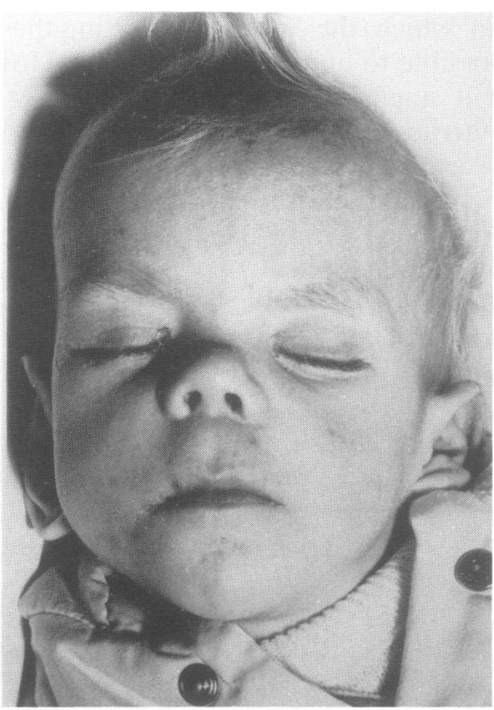

(b)
FIG 1 Craniofacial dysmorphism in two infants with reduced cictivity of dihydroxyacetonephosphate acyl transferase but normal peroxisomal $\beta$ oxidation. (a) Patient $I$ has $X$ linked dominant Conradi-Hünermann syndrome and (b) patient 2 has rhizomelic chondrodysplasia punctata.
Hünermann syndrome the patchy nature of the skin lesions and the asymmetry of the skeletal changes could be explained by Lyonisation. ${ }^{34}$ The craniofacial features in rhizomelic chondrodysplasia punctata are also affected by the extent to which CNS involvement leads to microcephaly.

Patients with Zellweger syndrome, 'mild Zellweger variants', and patients with infantile Refsum disease have a defect in the biogenesis of peroxisomes; the organelles are absent or virtually absent from all cells. Cell fusion studies indicate that the defect in infantile Refsum disease may be at the same gene locus as the defect in Zellweger syndrome. ${ }^{35}$ As a result of the absence of peroxisomes, the activity of DHAPAT (an enzyme which is normally bound to the peroxisomal membrane) is depressed and peroxisomal $\beta$ oxidation is impaired. The clinical features reflect to a considerable extent a summation of those found in defects of peroxisomal $\beta$ oxidation and those found in patients with chondrodysplasia punctata, but there is a wide spectrum in the expression of all of the features. Patients with classical Zellweger syndrome have very obvious dysmorphic features; the mild variants who survive into the second decade of life and those with infantile Refsum disease may have very subtle dysmorphology (fig 2). Whether the variability is a reflection of the ability of some patients to assemble a few peroxisomes or whether the effect of absent peroxisomes can be modified by other genetic or environmental factors remains uncertain.

Patients with neonatal adrenoleucodystrophy also have a defect in the biogenesis of peroxisomes. Cell fusion studies indicate that this is not identical to the defect in Zellweger patients. Kelley et $a l^{37}$ have suggested that the effect on peroxisomal $\beta$ oxidation may be different; whereas Zellweger fibroblasts show marked accumulation of saturated very long chain fatty acids and some accumulation of unsaturated VLCFA, neonatal ALD fibroblasts accumulate less saturated VLCFA and considerably less unsaturated VLCFA. A similar phenomenon is seen in the plasma of these patients. This fits to some extent with the postulated effects of impaired peroxisomal $\beta$ oxidation; neonatal ALD patients are less likely than Zellweger patients to show a retarded bone age and large fontanelle or cystic kidneys. However, both groups of patients show marked truncal hypotonia and defects of neuronal migration, particularly in the region of the brainstem and cerebellum. The impairment of plasmalogen synthesis may also be less marked in neonatal ALD than in Zellweger syndrome. This could explain the absence of chondral calcification and less obvious craniofacial dysmorphism in neonatal ALD.

Although in the above discussion we have drawn attention to the association between reduced plasmalogen synthesis and punctate epiphyseal calcification, Gilbert et $a l^{38}$ have listed a number of other conditions in which such calcification can occur, but in which impaired plasmalogen synthesis has not been found, for example, warfarin embryopathy. We could add to this list $\beta$ glucuronidase deficiency (R M Winter, P T Clayton, unpublished obser- 


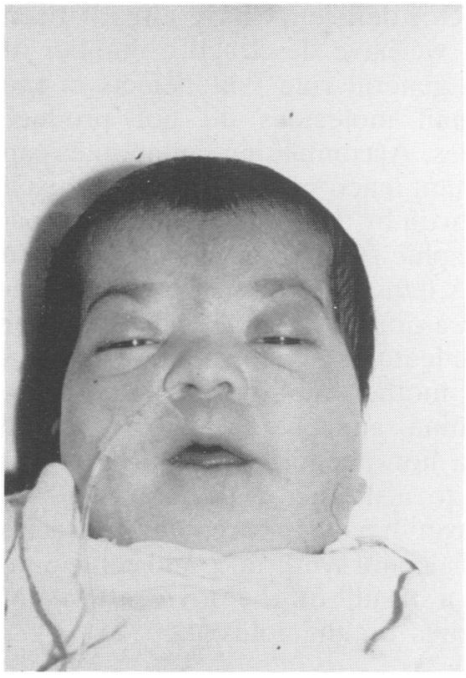

(a)

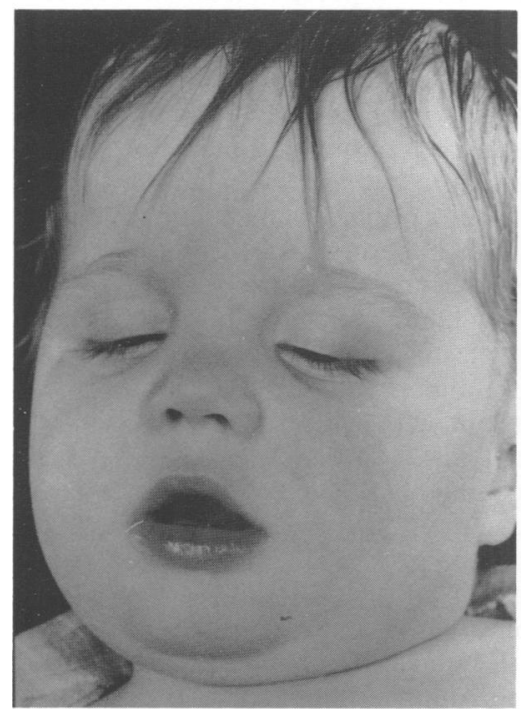

(d)

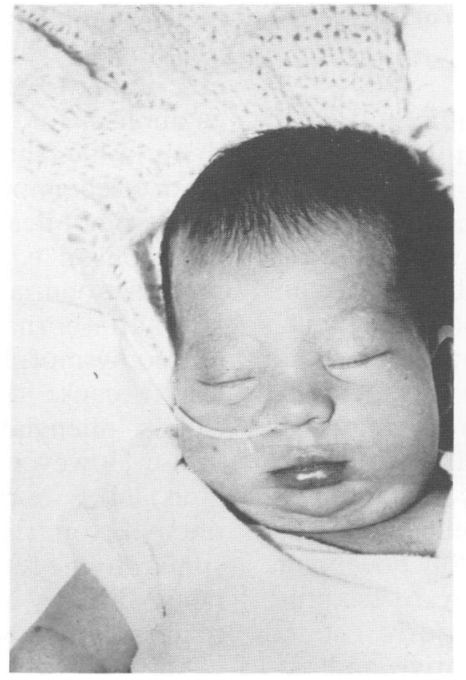

(b)

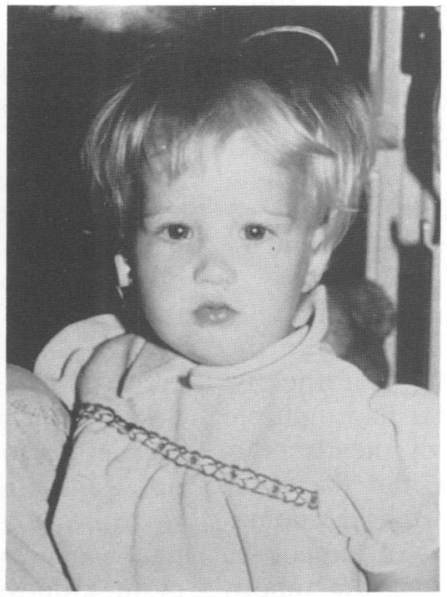

(e)

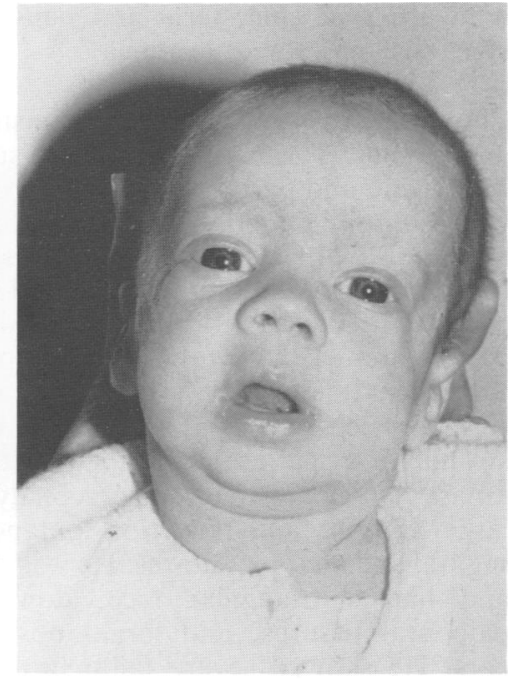

(c)

FIG 2 The spectrum of craniofacial dysmorphism in patients with absent peroxisomes. Patients 1 (a) and 2 (b) had classical Zellweger syndrome and patient 3 (c) had most of the features of classical ZS (see reference 36, patient AP) but has more subtle dysmorphic features. Patient $4(d)$ and his brother have many features of ZS but have survived beyond the age of two and 10 years respectively ('mild Zellweger variants'). (e) Patient $5\left(C B^{36}\right)$ had infantile Refsum disease.

vations), idiopathic neonatal hepatitis (E Naughten, 1987, personal communication), and a defect of peroxisomal $\beta$ oxidation ( $\mathrm{P} \mathrm{T}$ Clayton et al, unpublished data). One possible unifying explanation would be haemorrhage into the cartilage in utero perhaps because of hypoprothrombinaemia; hypo- prothrombinaemia could result from liver disease, from peroxisomal dysfunction, or from maternal warfarin therapy. Punctate epiphyseal calcification can also occur when the normal actions of thyroid hormone are prevented, for example, in familial resistance to thyroid hormone. ${ }^{1}$ 
A disorder of cholesterol and nonsterol isoprene synthesis: mevalonic aciduria ${ }^{3940}$

Cholesterol is an important constituent of cell membranes. The body's needs are supplied in part ty the diet and partly by synthesis from acetyl-CoA via 3-hydroxy-3-methylglutaryl-CoA and mevalonic acid. This pathway also supplies cells with nonsterol isoprenes, including ubiquinone and haem A (components of the respiratory chain), dolichol (which participates in the synthesis of glycoproteins), and isopentenyl adenine (a precursor of isopentenyl tRNA which participates in DNA replication).

The first patient described with mevalonate kinase deficiency had microcephaly, cataracts, a large anterior and posterior fontanelle, and wide sagittal and metopic sutures. He was dolichocephalic and had a triangular face, downward slanting palpebral fissures, and large, posteriorly rotated ears. He showed severe failure to thrive and was hypotonic but hyperreflexic. Radiology revealed a severely retarded bone age and a CT scan showed generalised atrophy of the brain. It is not possible to say whether the dysmorphic features in this patient were a consequence of impaired synthesis of cholesterol and nonsterol isoprenes.

\section{Disorders of pathways of metabolism of simple molecules which do produce dysmorphic features}

3-Hydroxyisobutyryl CoA deacylase deficiency ${ }^{41}$ is an inborn error which only affects one step in the catabolism of an amino acid (valine). Nevertheless, the one reported case of this disorder had multiple congenital malformations. Details of the dysmorphic facial features were not given, but the other abnormalities included multiple vertebral anomalies, tetralogy of Fallot, and agenesis of the corpus callosum. It has been postulated that the inborn error of valine metabolism leads to the accumulation of ethylacrylyl-CoA, that this compound can form conjugates with compounds containing free sulphydryl groups, and that this is the cause of the malformations. Methylacrylyl esters have been shown to be potent teratogens in the rat. ${ }^{42}$

Nyhan and Sakati ${ }^{40}$ have suggested that patients with methylmalonic acidaemia all have a similar facial appearance; this is not our impression from the 15 cases seen at The Hospital for Sick Children, Great Ormond Street. Some patients with a deficiency of the pyruvate dehydrogenase complex have had a high bossed forehead, anteverted nose, large ears, and dislocated hips with external rotation of the legs. ${ }^{40}$

The dysmorphic features of cystathionine synthetase deficiency, sulphite oxidase deficiency, and molybdenum cofactor deficiency have already been mentioned. Thus, we have described a number of $\overrightarrow{\bar{D}}$ exceptions to the general rule that defects in the $\frac{7}{0}$ catabolism of small molecules do not produce $\frac{\mathrm{C}}{0}$ dysmorphic features. Accumulating metabolites can $\overline{\bar{c}}$ often affect important macromolecules in the fetus if $\vec{\nabla}$ they are not dealt with by the placenta and maternal metabolism. A graphic illustration of this is seen in $\approx$ phenylketonuria. A fetus with (homozygous) phenyl- $\overrightarrow{0}$ ketonuria born to a normal (heterozygous) mother $\vec{\overrightarrow{ }}$ has no dysmorphic features or congenital malforma- $\vec{\sigma}$ ions because the mother rapidly metabolises the excess phenylalanine and metabolites from the fetus. However, a non-phenylketonuric (heterozy- iv gous) fetus born to a mother with (homozygous) iv phenylketonuria can have microcephaly, a facies iे similar to the fetal alcohol syndrome, and congenital $\mathrm{\omega}$

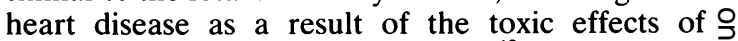
maternal metabolites on the fetus. ${ }^{43}$

Dysmorphic syndromes with biochemical abnormalities in which the precise metabolic defect is unknown

Some patients with glutaric aciduria type II have dysmorphic features. The first such patient ${ }^{404} 4$ had a large head, a large fontanelle, and widely spaced sutures. The ears were low set, the palpebral fissures small, and the inner canthal distance increased. The forehead was high and the nasal bridge low. The nose was short, the nares upturned, and the philtrum long. The hands showed bilateral simian creases and dysplastic nails. The infant had polycystic kidneys. Lehnert et $a l^{45}$ and Böhm et $a l^{46}$ described a patient with wide set eyes, prominent epicanthic folds, large, low set ears, a flattened nose, and a receding chin. The facial features of his sib were not commented upon, but both brothers had warty dysplasia of the cerebral cortex, poly- $\frac{3}{3}$ cystic kidneys, and intrahepatic biliary hypoplasia. The pattern of organic acids excreted in glutaric aciduria type II suggests that the defect affects $\frac{7}{0}$ flavine adenine dinucleotide (FAD) containing enzymes. In the non-dysmorphic patients, accu- N mulation of sarcosine suggested a defect affect- $N$ ing the transfer of electrons from mitochondrial FAD dependent dehydrogenases to coenzyme $Q$ in ${ }_{0}$ the respiratory chain. The cause of dysmorphic GA II has not yet been fully elucidated. Sarcosine 0 accumulation does not appear to occur. Thus, it is possible that the defect in this case affects glutaryl $\mathrm{CoA}$ and acyl $\mathrm{CoA}$ oxidases in the peroxisomes rather than the corresponding dehydrogenases in $\frac{\mathbb{D}}{\mathbb{D}}$ the mitochondria. Peroxisomal oxidases appear to $\frac{O}{\mathbb{Q}}$ be FAD dependent but it is thought that the $\stackrel{\square}{\varrho}$ electrons from the $\mathrm{FADH}_{2}$ are transferred to molecular oxygen (producing hydrogen peroxide) 
rather than to coenzyme $Q .{ }^{47}$ There are certainly similarities between the dysmorphic features of GA II and those of the peroxisomal defects.

Recently, Patton et $a l^{48}$ have described raised plasma and urinary 2-oxoglutarate levels in three patients with the DOOR syndrome (deafness, onycho-osteodystrophy, and mental retardation). Further studies will be needed before we can start to explain the relationship between the biochemical abnormality and the dysmorphic features in this instance.

The cause of hypercalcaemia in Williams syndrome also remains obscure; detailed studies have failed to show any definite abnormality of vitamin D metabolism ${ }^{49}$ but impaired calcitonin secretion has been reported in five children. ${ }^{50}$

\section{Future research}

It is clear that we have much to learn about the biochemistry of dysmorphism. The reactions which are likely to produce the most severe dysmorphic features (and may prove the most difficult to study) are those relating to the metabolism and mode of action of the substances that induce organogenesis in the embryo, the morphogens. The first vertebrate morphogen was identified in 1987: all-trans-retinoic acid is produced in posterior limb bud tissue in the chick and its concentration gradient induces the correct sequence of digits in the developing limb. ${ }^{51}$ Retinoic acid is a hydrophobic compound of relatively low molecular mass, characteristics which may allow it to pass from cell to cell without gap junctions or specialised receptors. ${ }^{52}$ It may be worthwhile to investigate the metabolism of this type of compound in patients with gross limb reduction defects.

Identification of an inborn error by metabolic studies sometimes makes it possible to localise the gene defect. Take, for example, hypophosphatasia. It is reasonable to assume that this disorder is caused by a mutation in the structural gene for alkaline phosphatase. Recently, the structural gene for the liver/bone/kidney form of alkaline phosphatase (ALPL) has been mapped to chromosome 1p34-p36-1. ${ }^{53}$ Since the ALPL cDNA detects a restriction fragment length polymorphism with the restriction endonuclease $B c l I$, it will be possible to undertake linkage studies in families with more than one child with hypophosphatasia to test the hypothesis that the mutation is at this site.

We thank Drs J V Leonard, D G Atherton, D B Grant, J Wilson, and E Brett for allowing us to publish photographs of the patients and we thank the families themselves. We are grateful to Professor Marcus Pembrey for his helpful comments.

\section{Note added in proof}

Further clues concerning the link between hypoprothrombinaemia and stippled epiphyses have emerged from studies on a boy with a coagulopathy and the phenotype of warfarin embryopathy. ${ }^{54}$ It is now well established that glutaric aciduria type II is usually the result of an inherited defect of electron transport flavoprotein (ETF) or ETF:ubiquinone oxidoreductase but that the dysmorphic patients do not have this defect. ${ }^{55}$

\section{References}

' Stanbury JB, Wyngaarden JB, Fredrickson DS, Goldstein JL, Brown MS, eds. The metabolic basis of inherited disease. 5th ed. New York: McGraw-Hill, 1983.

2 Benson PF, Fensom AH. Genetic biochemical disorders. Oxford: Oxford University Press, 1985.

${ }^{3}$ Cheah KSE. Collagen genes and inherited connective tissue disease. Biochem J 1985;229:287-303.

+ Sykes B. The molecular genetics of collagen. BioEssays 1985;3: 112-7.

5 Aitchison K, Ogilvie D, Honeyman M, Thompson E, Sykes B. Homozygous osteogenesis imperfecta unlinked to collagen I genes. Hum Genet 1988:78:233-6.

6 Steinmann B, Tuderman L, Peltonen L, Martin GR, McKusick VA, Prockop DJ. Evidence for a structural mutation of procollagen type $I$ in a patient with Ehlers-Danlos syndrome type VII. J Biol Chem 1980;255:8887-93.

7 Prockop DJ, Kivirikko KI. Heritable diseases of collagen. $N$ Engl J Med 1984;311:376-86.

^ Eyre DR, Shapiro FD, Aldridge JF. A heterozygous collagen defect in a variant of Ehlers-Danlos syndrome type VII. J Biol Chem 1985;260:11322-9.

9 Cole WG, Chan D, Chambers GW, Walker ID, Bateman JF. Deletion of 24 amino acids from the pro- $\alpha 1$ (I) chain of type I procollagen in a patient with Ehlers-Danlos syndrome type VII. J Biol Chem 1986;261:5496-503.

10 Cole WG, Evans R, Sillence DO. The clinical features of Ehlers-Danlos syndrome type VII due to a deletion of 24 amino acids from the pro $\alpha 1$ (I) chain of type I procollagen. $J$ Med Genet 1987;24:698-701.

1 McKusick VA. Mendelian inheritance in man. Catalogs of autosomal dominant, autosomal recessive and $X$-linked phenotypes. (No 22541). 7th ed. Baltimore: Johns Hopkins University Press, 1986.

12 Pope FM, Martin GR, Lichtenstein JR, et al. Patients with Ehlers Danlos syndrome type IV lack type III collagen. Proc Natl Acad Sci USA 1975;72:1314-7.

13 Pope FM, Nicholls AC, Dorling J, Webb J. Molecular abnormalities of collagen: a review. J $R$ Soc Med 1983;76:1050-62.

14 Pinnell SR, Krane SM, Kenzora JE, Glimcher MJ. Heritable disorder with hydroxylysine-deficient collagen. Hydroxylysinedeficient collagen disease. $N$ Engl J Med 1972;286:1013-20.

15 Anderson LL, Oikarinen AJ, Ryhänen L, Anderson CE, Uitto J. Characterisation and partial purification of a neutral protease from the serum of a patient with autosomal recessive pulmonary emphysema and cutis laxa. J Lab Clin Med 1985;105:537-46.

16 Byers PH, Siegel RC, Holbrook KA, Narayanan AS, Bornstein P, Hall JG. X-linked cutis laxa. Defective cross-link formation in collagen due to decreased lysyl oxidase activity. $N$ Engl J Med 1980;303:61-5.

17 Wadman SK, Duran M, Beemer FA, et al. Absence of hepatic molybdenum cofactor: an inborn error of metabolism leading to a combined deficiency of sulphite oxidase and xanthine dehydrogenase. J Inherited Metab Dis 1983;6(suppl 1):78-83.

${ }^{18}$ Elsas LJ, Endo F, Strumlauf E, Elders J, Priest JH. Lep- 
rechaunism: an inherited defect in a high-affinity insulin receptor. Am J Hum Genet 1985;37:73-88.

${ }^{19}$ McLean RH, Hoefnagel D. Partial lipodystrophy and familial C3 deficiency. Hum Hered 1980;30:149-54.

211 Watts RWE, Gibbs DA. Lysosomal storage diseases. London: Taylor and Francis, 1986.

21 Cooper A, Sardarwalla IB, Roberts MM. Human $\beta$ mannosidase deficiency. $N$ Engl J Med 1986;315:1231.

22 Wenger DA, Sujansky E, Fennessey PV, Thompson JN. Human $\beta$-mannosidase deficiency. $N$ Engl $J$ Med 1986;315: 1201-5.

23. Schutgens RBH, Heymans HSA, Wanders RJA, van den Bosch $H$, Tager JM. Peroxisomal disorders: a newly recognised group of genetic diseases. Eur J Pediatr 1986;144:430-40.

${ }^{24}$ Danpure CJ. Jennings PR, Watts RWE. Enzymological diagnosis of primary hyperoxaluria type 1 by measurement of hepatic alanine:glyoxylate aminotransferase activity. Lancet 1987;i: 289-91.

25 Lazarow PB. The role of peroxisomes in mammalian cellular metabolism. J Inherited Metab Dis 1987:10(suppl 1):11-22.

26 Wanders RJA, van Roermund CWT, van Wijland MJA, et al. Peroxisomal fatty acid $\beta$-oxidation in relation to the accumulation of very long chain fatty acids in cultured skin fibroblasts from patients with Zellweger syndrome and other peroxisomal disorders. J Clin Invest 1987;80:1778-83.

27 Moser HW, Moser AE, Singh I, O'Neill BP. Adrenoleukodystrophy: survey of 303 cases: biochemistry, diagnosis and therapy. Ann Neurol 1984:16:628-41.

${ }^{28}$ Poll-Thé BT, Roels F, Ogier F, et al. Pseudo-neonatal adrenoleukodystrophy: a new peroxisomal disorder with giant peroxisomes and a specific defect of acyl-CoA oxidase. Am J Hum Genet (in press).

29 Goldfischer S, Collins J, Rapin I, et al. Pseudo-Zellweger syndrome: deficiencies in several peroxisomal oxidative activitics. J Pediatr 1986;108:25-32.

3) Schram AW, Goldfischer S, Van Roermund CWT, et al. Human peroxisomal 3-oxoacyl-coenzyme A thiolase deficiency. Proc Natl Acad Sci USA 1987:84:2494-6.

31 Clayton PT, Lake BD, Hjelm M, et al. Bile acid analyses in pscudo-Zellweger syndrome: clucs to the defect in peroxisomal B-oxidation. J Inherited Metab Dis 1988:11(suppl 2) (in press).

32 Heymans HSA, Oorthuys JWE, Nelck G, Wanders RJA, Schutgens RBH. Rhizomelic chondrodysplasia punctata: another peroxisomal disorder. $N$ Engl J Med 1985;313:187-8.

33 Holmes RD, Wilson GN, Hajra AK. Peroxisomal enzyme deficiency in the Conradi-Hünermann form of chondrodysplasia punctata. $N$ Engl J Med 1987:316:1608.

${ }^{34}$ Mueller RF, Crowle PM, Jones RAK. Davison BCC. X-linked dominant chondrodysplasia punctata: a case report and family studics. Am J Med Genet 1985:20:137-44.

35 Wanders RJA, Saclman D. Heymans HSA, et al. Genetic relation between the Zellweger syndrome, infantile Refsum's discase and rhizomelic chondrodysplasia punctata. $N$ Engl $J$ Med 1986;314:787-8.

${ }^{36}$ Clayton PT, Lake BD. Hall NA, Shortland DB, Carruthers RA. Lawson AM. Plasma bilc acids in patients with peroxisomal dysfunction syndromes: analysis by capillary gas chromatography-mass spectrometry. Eur J Pediatr 1987:146:166-73.

${ }^{37}$ Kelley RI. Datta NS, Dobyns WB, et al. Nconatal adrenoleukodystrophy: new cases, biochemical studies and differentiation from Zellweger and related peroxisomal polydystrophy syndromes. Am J Med Genet 1986:23:869-901.
${ }^{38}$ Gilbert EF, Opitz JM, Spranger JW, Langer LO, Wolfson JJ: Viseskull C. Chondrodysplasia punctata-rhizomelic form. Patho logical and radiological studies of three infants. Eur J Pedia $F^{+}$ 1976;123:89-109.

39 Hoffmann G, Gibson KM, Brandt IK, Bader PI, Wappner RS우 Swectman L. Mevalonic aciduria-an inborn error of choles $\bar{c}$ terol and nonsterol isoprene biosynthesis. $N$ Engl $J$ Me 1986;314:1610-4.

4) Nyhan WL. Sakati NA. Diagnostic recognition of genetiछ) disease. Philadelphia: Lea \& Febiger, 1987.

+ Brown GK, Hunt SM, Scholem R, et al. $\beta$-Hydroxyisobutyry $\vec{P}$ CoA deacylase deficiency: a defect in valine metabolism associated with physical malformations. Pediatrics 1982;70 $\overrightarrow{\mathrm{w}}$ 532-8.

t2 Singh AR, Lawrence WH, Autian J. Embryonic-fetal toxicit and teratogenic effects of a group of methacrylate esters in ratso J Dent Res 1972;51:1632-8.

4.3 Lipson A, Yu JS, O'Halloran MI, Williams R. Alcohol and phenylketonuria. Lancet $1981 ; \mathbf{i}: 717-8$.

4 Swectman L, Nyhan WL, Trauner DA, Merritt TA, Singh Mô Glutaric aciduria type II. J Pediatr 1980;96:1020-6.

45 Lehnert W, Wendel U, Lindenmaier S, Böhm N. Multiple acyf CoA dehydrogenation deficiency (glutaric aciduria type II). congenital polycystic kidneys, and symmetric warty dysplasia of the cerebral cortex in two brothers. I. Clinical, metabolic an biochemical findings. Eur J Pediatr 1982;139:56-9.

th Böhm N, Uy J, Kiessling M. Lehnert W. Multiple acyl-Co $\overrightarrow{A O}$ dehydrogenation deficiency (glutaric aciduria type II), con $0^{\circ}$ genital polycystic kidneys, and symmetric warty dysplasia of the cerebral cortex in two newborn brothers. II. Morphology an pathogenesis. Eur J Pediatr 1982;139:60-5.

47 Vamecq J, Van Hoof F. Implications of a peroxisomal enzym in the catabolism of glutaryl-CoA. Biochem J 1984:221:203-11O

${ }^{48}$ Patton MA, Krywawych S, Winter RM, Brenton DB, Baraitseon M. DOOR syndrome (deafness, onycho-osteodystrophy an $\$$ mental retardation): elevated plasma and urinary 2-oxoglutarate in threc unrelated patients. Am J Med Genet 1987:26:207-15.음

${ }^{49}$ Martin NDT, Snodgrass GJAI, Cohen RD, et al. Vitamin metabolites in idiopathic infantile hypercalcacmia. Arch $D$ 氶 Child 1985:60:1140-3.

5) Culler FL. Jones KL, Deftos LJ. Impaired calcitonin secretion in patients with Williams syndrome. J Pediatr 1985;107:720-3.3.

5) Thaller C. Eichele G. Identification and spatial distribution retinoids in the developing chick limb bud. Nature 1987;327\% 625-8.

52 Slack JMW. We have a morphogen! Nature 1987;327:553-4.

53 Weiss M, Smith M, Griffin C, et al. Assignment of the gen encoding the liver/bone/kidney form of alkaline phosphatase ALPL to the region 1p34-p36-1. HGM9. Cytogenet Cell Geng (in press).

54 Pauli RM, Lian JB, Mosher DF, Suttie JW. Association congenital deficiency of multiple vitamin K-dependent coagu lation factors and the phenotype of the warfarin embryopatho Am J Hum Genet 1987;41:566-83.

55 Goodman SI, Frerman FE, Loehr JP. Recent progress in understanding glutaric acidemias. Enzyme 1987:38:76-79.

Correspondence and requests for reprints to Dr E M Thompson, Mothercare Department of Paediatrio Genetics, Institute of Child Health, 30 Guilfor $\widetilde{\Phi}$ Street, London WC1N 1EH. 